\title{
Self-Pruning Broadcasting for Mobile Ad Hoc Networks
}

\author{
Wilson Woon \\ Dept. of Electrical and Electronic Engineering \\ Faculty of Engineering \\ The University of Hong Kong \\ Pokfulam, Hong Kong \\ Email: thwoon@eee.hku.hk
}

\author{
Kwan L Yeung \\ Dept. of Electrical and Electronic Engineering \\ Faculty of Engineering \\ The University of Hong Kong \\ Pokfulam, Hong Kong \\ Email: kyeung@eee.hku.hk
}

\begin{abstract}
Broadcasting is a process of delivering a message to all nodes in a network. While it is important to ensure that all nodes get a copy of the broadcast message, minimizing the number of sending nodes is equally important especially in resource-constrained wireless networks. Existing broadcasting protocols based on self-pruning are ineffective in achieving these objectives. Therefore this paper proposes two protocols based on simple timer mechanisms to prioritize broadcasting of messages such that node with most uncovered neighbors rebroadcast first. Additionally a timer suppression mechanism is proposed to further enhance the effectiveness of the broadcasting protocol. Compared with an existing protocol, extensive simulation experiments confirm that the proposed protocols achieve better performance.
\end{abstract}

\section{INTRODUCTION}

Mobile Ad Hoc Networks (MANETs) are popular wireless networks that received tremendous attentions over the last decade due to their inherent flexibility and simple implementation. They are created on the fly without the need for any centralized networks. All nodes are regarded as homogeneous and distributed across the networks without any fixed topology. A typical MANET may contain hundreds to thousands of inexpensive, small-sized, and battery-powered nodes collaborating with each other to perform a network function. Due to these characteristics, MANET is widely used in areas like military and surveillance, vehicular communications, health care, environmental monitoring, forest fire detection, and many more where seamless connections among nodes are important.

Network-wide broadcasting, or simply broadcasting, is a process of delivering a message to all nodes in a network. It is regarded as an essential mechanism that drives the success of MANET, where broadcasting protocol plays an important role in establishing routes for on demand routing protocols and building routing tables for table-driven routing protocols.

Since broadcasting is an integral part of MANET, good design of a broadcasting algorithm is essential. While it is important to ensure that all nodes get a copy of the broadcast message, minimizing the number of sending nodes is equally important since any saved broadcast could prolong the lifetime of nodes that are battery powered, lower channel utilization, and reduce packet collisions in congested networks. There are numerous broadcasting protocols that address these issues.
They can be classified into self-pruning and deterministic (and a review is given in Section 2). This paper focuses on the former due to its simple distributed control and easy implementation. In particular, this research is motivated by the timer mechanism of Scalable Broadcasting Algorithm (SBA) [1]. SBA is an example of self-pruning protocol that sets the broadcast timer of a node based on the number of neighbors it has. The more neighbors a particular node has, the earlier it gets to timeout and rebroadcast. This method of prioritizing broadcasting is unique and rarely explored. The mechanism adopted by SBA is, however, not optimized and can be further improved. In this paper two new protocols, namely Enhanced SBA (E-SBA) and Enhanced SBA+ (E-SBA+) are proposed to address the deficiencies of SBA protocol.

This paper is organized as follows. Section 2 gives a survey of existing broadcasting protocols based on self-pruning. This is followed by detail descriptions of the two proposed broadcasting protocols in Section 3. Section 4 presents the simulation settings while the results are given in Section 5. Finally, Section 6 concludes the paper.

\section{Review of Self-Pruning Broadcasting PROTOCOLS}

Broadcasting based on self-pruning gives each node the freedom to decide whether or not to rebroadcast a packet. In contrast, the deterministic approach requires the sending node to select one or more nodes to perform the broadcasting. This paper focuses on the self-pruning approach which can be further divided into simple flooding, probabilistic, area-based, neighbor-knowledge, and timer-based.

Simple flooding is the most straightforward broadcasting protocol. Each node simply rebroadcasts a unique message at most once. This ensures high delivery ratio but often causes inefficient use of nodal resources and unnecessary bandwidth consumption. Besides, each node needs to keep track the message IDs. Due to its simplicity, flooding is widely used in various unicast routing protocols $[4,5,6]$.

One simple improvement to flooding is called probabilistic broadcasting [2]. Each unique message is rebroadcast once based on certain fixed probability. This method works well in 
dense networks where multiple nodes cover the same transmission range. Therefore, not all nodes need to rebroadcast. However, in a sparse network probabilistic flooding cannot ensure all nodes receive the message. Counter based [2] is another variant to probabilistic broadcasting where each node uses a counter to keep track of the number of times the same message is received. If the counter exceeds a threshold, the broadcasting is cancelled. Therefore, setting the appropriate threshold value is perhaps the most critical. The work by Tseng et. al. [2,3] involved fine-tuning the threshold against various types of scenarios.

Area based methods [2] require each node to evaluate the additional area that it can cover when rebroadcast. If the evaluation result exceeds a threshold, then the message is sent for rebroadcasting. There are two approaches in calculating the additional coverage area, distance-based and location-based. The latter assumes each node is equipped with a Global Positioning System (GPS) receiver to obtain precise location while the former uses signal strength to approximate the distance between sending and receiving nodes. The use of GPS receivers adds additional cost and energy consumption which could be expensive especially in a MANET where the population could span from hundreds to thousands of nodes. The problem with location-based approach is that signal strength does not give accurate location information and is susceptible to interferences.

Neighbor-knowledge scheme $[2,7,8,9,10,11]$ relies on neighborhood information obtained via periodic HELLO packet exchanges. Each HELLO packet contains the identity of the sender and its neighbors. This message is used to maintain up-to-date knowledge of immediate and 2-hop neighbors (neighbors of immediate neighbors). Such information is readily available in some MANET routing protocols. Then a node could easily identify its uncovered neighbors as nodes that are not immediate neighbors of the sender.

SBA is an example of timer-based broadcasting protocol. It has all the features of neighbor-knowledge scheme but with an additional timer mechanism. This mechanism is the main factor that makes it more effective than many existing selfpruning protocols. The basic idea of SBA is to allow nodes with most neighbors to broadcast earlier by having shorter "backoff" periods or delays. SBA is designed to broadcasting towards the densest area in the shortest time; this could reduce the number of broadcasting required. Each node $A$ that receives a message and has at least one uncovered neighbor will set a delay timer $T$ based on the following ratio:

$$
\begin{gathered}
T 0=\frac{1+d_{\max }(A)}{1+d(A)} \\
T=U(\Delta \times T 0)
\end{gathered}
$$

where $d(A)$ is the degree of node $A$ (the number of neighbors node $A$ has) and $d_{\max }(A)$ is the maximum degree of any neighbor of $A . \Delta$ is a small constant delay and $U(X)$ is a function that returns a uniformly distributed random number between 0 and $X$. The purpose of $\Delta$ is to give sufficient time for nodes to receive redundant messages. This also reduces the possibility of packet collision when a contention-based medium access control (MAC) protocol such as the IEEE 802.11 is used. Since this paper does not assume the use of any MAC protocol, the best value of $\Delta$ depends only on if the time reserved for receiving redundant messages is sufficient. The optimal values for $\Delta$ will be examined in Section 4.

While SBA is an effective protocol, there are some deficiencies. First, the timer setting in (1) and (2) actually does not allow nodes with most neighbors to broadcast earlier. Consider two nodes with equal number of 1-hop neighbors. Node with less 2-hop neighbors will rebroadcast first. Another problem is that the timer ratio takes all neighbors into consideration even though they have already received the message.

\section{Proposed Self-Pruning Broadcasting PROTOCOLS}

Two new self-pruning broadcasting protocols are proposed in this paper, namely Enhanced SBA (E-SBA) and Enhanced $\mathrm{SBA}+(\mathrm{E}-\mathrm{SBA}+)$.

\section{A. Enhanced SBA (E-SBA)}

E-SBA solves the problems of SBA by simply modifying the original delay timer, $T$. Assume node $A$ receives a message from node $B$. Node $A$ sets its rebroadcast delay timer as follows:

$$
\begin{gathered}
T 0=\frac{1}{d^{*}(A)}+\frac{1}{d_{\max }^{*}(A)} \\
T=U(\Delta \times T 0)
\end{gathered}
$$

where $d^{*}(A)$ is the updated degree of node $A$ and $d_{\text {max }}^{*}(A)$ is the updated maximum degree of any neighbor of node $A$. The former can be expressed mathematically as the sum of nodes in $N(A) \cap N(B) \cap\{B\}$ where $N(A)$ and $N(B)$ are sets of immediate neighbors of nodes $A$ and $B$ respectively. Unlike SBA, the proposed design of $T 0$ ensures nodes with more uncovered first- and second-hop neighbors rebroadcast first, whereas covered nodes are not considered. Assume nodes $A$ and $B$ receive the broadcast message from some node at the same time and $d(B)>d(A)$. Nodes $A$ and $B$ are then competing to rebroadcast the message. In SBA, node $B$ will rebroadcast first (with higher probability) even though it may have more covered neighbors than node $A$, i.e. $d^{*}(A)>d^{*}(B)$. By allowing node $B$ to rebroadcast first could potentially direct traffic among covered nodes (instead of uncovered) thus having more nodes rebroadcast. The delay timer, $T$, is assumed to be sensitive enough to take on floating point values so as to differentiate the broadcast timers.

To further elaborate on the idea behind E-SBA, the example in Fig. 1 is considered. Assume nodes 1 and 2 receive a message from node 0 and nodes 5 and 6 have been covered (by nodes not shown). In SBA, node 2 will rebroadcast first even though it has only three (one first-hop neighbor and two 
second-hop neighbors) uncovered nodes compared with node 1 which has four (two first-hop neighbors and second-hop neighbors each). E-SBA will ensure that node 1 rebroadcast first thus saving the broadcasting by node 2 .

The broadcasting procedure of E-SBA is summarized below:

Any node $A$, that receives a broadcast message, $m$, from sender $B$, will perform the following steps:

1) If $N(A) \subseteq N(B) \cup\{B\}$, then no rebroadcast need to be performed since all neighbors of $A$ have been covered by $B$. The subsequent duplicated messages will be discarded.

2) If message $m$ is received for the first time, then establish a covered set $C$ to store all covered nodes or more formally $C(A, m)=N(B) \cup\{B\}$. Based on the updated degree of node $A$, i.e. $d^{*}(A)$, determine $d_{\max }^{*}(A)$. Initialize the countdown timer to $T$ using (3).

3) During timer countdown, any duplicated message will be discarded and covered nodes set will be updated, i.e. $C(A, m)=C(A, m) \cup N(B) \cup\{B\}$

4) After the delay timer expires and $N(A) \subseteq C(A, m)$, node $A$ is inhibited from rebroadcast. Otherwise the message is rebroadcast.

In order to retain the simplicity of the proposed protocol, the initial timer value is set once, that is during the first time a particular message $m$ is received. Just like existing neighbor-knowledge and timer-based broadcasting techniques, E-SBA considers neighbors up to two hops only. Within 1-hop, there is almost no uncertainty in $d^{*}(A)$. However, $d_{\text {max }}^{*}(A)$ obtained from Step 2 above may incur some uncertainties. For example in Fig. 1, nodes 1 and 2 will not know that their 2-hop neighbors, i.e. nodes 7 and 8 have been covered by nodes 11 and 9 respectively. This problem persists not only in self-pruning approach but also in deterministic broadcasting protocols that involve multi-hop neighborhood information. A simple solution is to send an acknowledgement packet for every broadcast message received. This is highly undesirable because it causes high packet overhead and collisions. A more practical solution is to give certain weighting factor on $d_{\text {max }}^{*}(A)$. However, simulation studies show that this does not have much impact on the overall performance. This could be due to the uncertainty at 2-hop is not obvious enough. On the other hand, extending the protocol beyond 2-hop is possible. But the uncertainties of the neighborhood information become more pronounced, which can easily offset the potential gain in performance.

\section{B. Enhanced $S B A+(E-S B A+)$}

Thus far, E-SBA has solved the two fundamental problems with SBA. Since the original design objective of SBA is to quickly cover as many nodes as possible by allowing nodes with more neighbors to rebroadcast earlier, the example in Fig. 1 shows that existing mechanism is ineffective. There are 10 nodes in Fig. 1 with node 0 as the source node. Nodes 1 and 2 will start their respective delay timers to rebroadcast the message from node 0 . Node 2 will timeout first since it

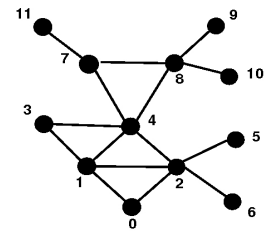

Fig. 1. A MANET with 12 nodes

has slightly more neighbors to cover than node 1 . Now nodes 4 and 1 are competing with each other to rebroadcast. Since the timer of node 1 has started, it will have the priority to rebroadcast earlier even though node 4 has more uncovered neighbors. Thus, the broadcasting by node 1 is redundant.

Notice that this problem could be solved by preventing node 1 from sending earlier than node 4. Therefore in E-SBA+, we propose to restart the timer of node 1 . That is whenever a node receives a rebroadcast message it knows that it has lower priority to retransmit. It must then suppress its timer by resetting it according to the new uncovered neighborhood information. This mechanism is known as timer suppression. Since node 4 has more neighbors than node 1 , it will cover nodes 3,7 , and 8 , thus saving the broadcasting by node 1 .

$\mathrm{E}-\mathrm{SBA}+$ is formally defined below:

Any node, say $A$, that receives a broadcast message, $m$, from sender $B$, will perform the following steps:

1) If $N(A) \subseteq N(B) \cup\{B\}$, then no rebroadcast need to be performed since all neighbors of $A$ has been covered by $B$. The subsequent duplicated messages will be discarded.

2) If message $m$ is received for the first time, then establish a covered set $C$ to store all covered nodes or more formally $C(A, m)=N(B) \cup\{B\}$. Based on the updated degree of node $A$, i.e. $d^{*}(A)$, determine $d_{\text {max }}^{*}(A)$. Initialize the countdown timer to $T$ using (3).

3) During timer countdown, any duplicate message will be discarded and covered nodes set will be updated, i.e. $C(A, m)=C(A, m) \cup N(B) \cup\{B\}$. Delay timer will be reset according to the latest uncovered neighborhood information.

4) After the delay timer expires and $N(A) \subseteq C(A, m)$ node $A$ is inhibited from rebroadcast. Otherwise the message is rebroadcast.

In addition to inheriting all the features of E-SBA, E$\mathrm{SBA}+$ introduces a timer suppression mechanism that delays the rebroadcasting of nodes with lower priority to enable nodes that rebroadcast earlier to cover as many nodes as possible (including uncovered neighbors of suppressed nodes). However not all suppressed nodes with be relieved from rebroadcasting. Assume node 3 of Fig. 1 is not within range of node 4 . Node 1 will be suppressed by node 4 and will eventually timeout to cover node 3 .

Theorem 1: A node will restart its timer at most $n-1$ times, where $n$ is the number of neighbors of a receiving node.

Proof: From the proposed E-SBA+ a node will restart 
TABLE I

COMMON SimUlation PARAMETERS

\begin{tabular}{|c||c|}
\hline \multicolumn{1}{|c||}{ Simulation Parameter } & Value \\
\hline Simulator & NS2.33 [12] \\
\hline Bandwidth & $11 \mathrm{Mbps}$ \\
\hline Network Size & $1000 \times 1000$ meters \\
\hline Transmission Range & 250 meters \\
\hline Node Max. IFQ Length & 50 \\
\hline Data Packet Size & 64 bytes payload \\
\hline Simulation Duration & 50 seconds \\
\hline HELLO Interval & 1 second \\
\hline No. of Trials & 20 \\
\hline Confidence Interval & $95 \%$ \\
\hline
\end{tabular}

its delay timer whenever it receives a message from one of its neighbors. It is easy to see that the worst case scenario is when a node has the lowest priority to broadcast among all its neighbors except the source/sender. That is $n-1$. However, our simulation study shows that nodes restart their timers on average three times. It is also easy to see that the best case is when all neighbors have been covered by a previous broadcasting.

Corollary 1: All suppressed timers will expire eventually.

Proof: From Theorem 1, there is a limit to the number of times a node will restart its timer.

Theorem 2: In a collision-free network, E-SBA+ can achieve $100 \%$ delivery upon termination of broadcasting.

Proof: By contradiction suppose there is at least one node that has not received the message after termination of the broadcast operation. Consider the following set

$\lambda=\left\{\left(N_{X}, N_{Y}\right) \mid N_{X}\right.$ and $N_{Y}$ are neighbors, $N_{X}$ has received the message while $N_{Y}$ has not

Suppose $N_{S}$ is the source node while $N_{D}$ is the node that has not received the message from $N_{S}$. Assuming the network is connected, there is a path between $N_{S}$ and $N_{D}$. Consider two neighbors, $N_{A}$ and $N_{B}$ along the path of $N_{S}$ and $N_{D}$ such that $N_{A}$ has received the message while $N_{B}$ has not. Consequently, $\left(N_{A}, N_{B}\right) \in \lambda$, thus $\lambda \neq \emptyset$. As a result,

$\exists\left(N_{A}, N_{B}\right) \in \lambda$ such that $\forall\left(N_{X}, N_{Y}\right) \in \lambda: N_{X}$ is being

suppressed from broadcast

However this contradicts with Corollary 1 and the theorem is proved.

\section{Simulation Settings}

The primary objective of this simulation study is to evaluate the effectiveness of the proposed E-SBA and E-SBA+ by comparing with SBA. We conduct two studies, Algorithm Effectiveness and Mobile Networks. Table 1 summarizes the general simulation settings.

As mentioned in Section 2, finding the appropriate value for $\Delta$ is important to give sufficient time for each node to receive redundant messages. Based on (1) and (3), it is obvious that the timer value for SBA is always greater than 1s while ESBA and E-SBA+ are always less than 1s. Therefore, SBA would incur higher delay in its rebroadcasting, thus having more opportunity for receiving redundant messages compared with E-SBA and E-SBA+. In the first part of this study, the SBA protocol is extended to incorporate the proposed timer suppression mechanism. An evaluation is conducted to compare the original SBA with its extended version to determine how effective the timer suppression mechanism is. In the second part, SBA and the proposed protocols are evaluated using randomly chosen $\Delta$ values of $0.3333,0.01$, and 0.0001 which represent decreasing order of timer delays. That is 0.3333 and 0.0001 would incur the highest and lowest broadcast delay respectively. The network size is between 20 to 200 nodes and the broadcast origination rate is 1 pps. Even though this study would clearly favor SBA, it is important to evaluate to what extent the $\Delta$ values would affect the performances of the proposed protocols. For a fairer comparison, the third part of this study attempts to identify the $\Delta$ values that would incur comparable broadcast latency for all protocols. In order to achieve this, a network size of 100 nodes and broadcast origination rate of 1 packet per second (pps) is setup to simulate various $\Delta$ values. Theoretically, more nodes will rebroadcast when small $\Delta$ values are used. The overall objective of Study 1 is to evaluate the core algorithms of SBA, E-SBA and E-SBA+ in a static network with Null MAC.

Study 2 is known as Mobile Networks. The focus is on evaluating the ability of each protocol in reacting to node mobility. This study is based on a steady-state random waypoint mobility model [13] with zero pause time. Nodes are randomly chosen to move at speeds between 1 to 20 meters per second $(\mathrm{m} / \mathrm{s})( \pm 10 \%)$ in a network of 100 nodes. Similar to Study 1, the protocols are evaluated using various network sizes between 20 to 200 , and $\Delta$ values of $0.3333,0.01$, and 0.0001 .

The following performance metrics were used:

- Number of Rebroadcasting Nodes: total number of nodes retransmitting a packet (excluding the source node).

- Packet Delivery Ratio (PDR): ratio between the number of packets that are successfully received and the total number of packets sent.

- Broadcast Latency: time required for the slowest/last node to get a successful packet.

\section{Results}

Simulation results of Study 1 - Algorithm Effectiveness is presented. The effectiveness of the timer suppression mechanism is presented first. Fig. 3 compares SBA with an extended version of it. The difference between SBA and the extended version is the latter incorporates the timer suppression mechanism. (In this case, timer suppression does not involve the recalculation of the initial timer value.) A $\Delta$ value of 0.01 is selected for both protocols. Clearly the extended version performs better than the original protocol which proves the effectiveness of the proposed timer suppression mechanism.

As mentioned previously, SBA will always incur longer broadcasting delay than the proposed protocols. This is shown in Fig. 4. Therefore, it is not surprising that SBA outperforms 


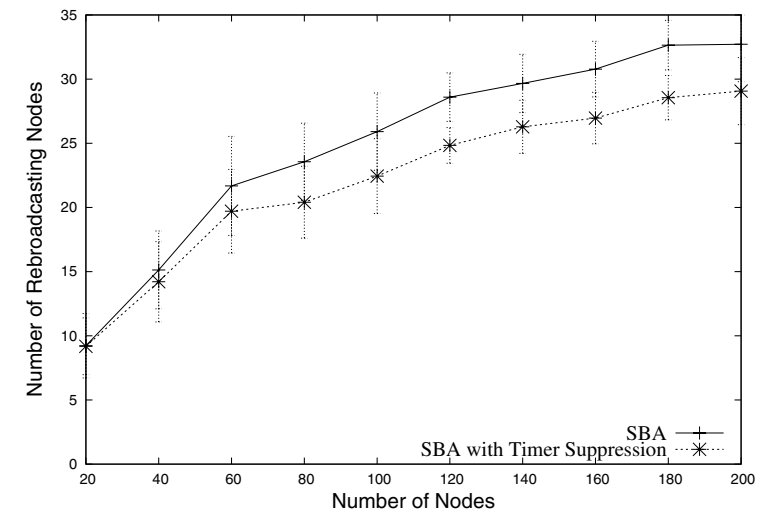

Fig. 2. SBA vs SBA with Timer Suppression

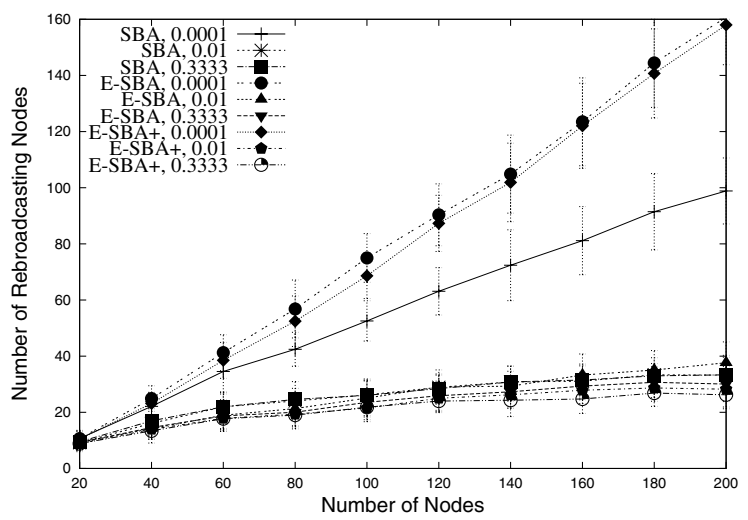

Fig. 3. Number of Rebroadcasting Nodes vs. Number of Nodes using various $\Delta$ values

E-SBA and E-SBA+ in Fig. 3 with $\Delta=0.0001$. However, with larger $\Delta$ values such as 0.3333 , the proposed protocols require the least number of nodes to cover the entire network. Between E-SBA and E-SBA+, the latter emerges as the best performer. In most situations, SBA has better opportunity to eliminate redundant rebroadcasting. However, when the duration of broadcasting delay is sufficient, the effectiveness of the proposed protocols outperforms SBA. From this experiment, one would predict that the delay incurred by E-SBA+ should be significantly higher than E-SBA due to the timer suppression mechanism which further delays the rebroadcasting of packets. However, Fig. 4 shows otherwise and the increase in delay is not substantial.

Setting the appropriate $\Delta$ values for each protocol is important to have a fair comparison on broadcast latency. Fig. 5 shows that SBA, E-SBA, and E-SBA+ should adopt 0.1428, 0.5 , and 0.3333 respectively as their $\Delta$ values. By looking at their performances based on these $\Delta$ values, it is clear from Fig. 6 that E-SBA+ is the most effective protocol, followed by E-SBA and SBA. This shows that the two prposed timer settings are effective in reducing the number of rebroadcasting nodes even though there may exist inaccuracies in the 2hop neighborhood information as discussed in Section 3 .

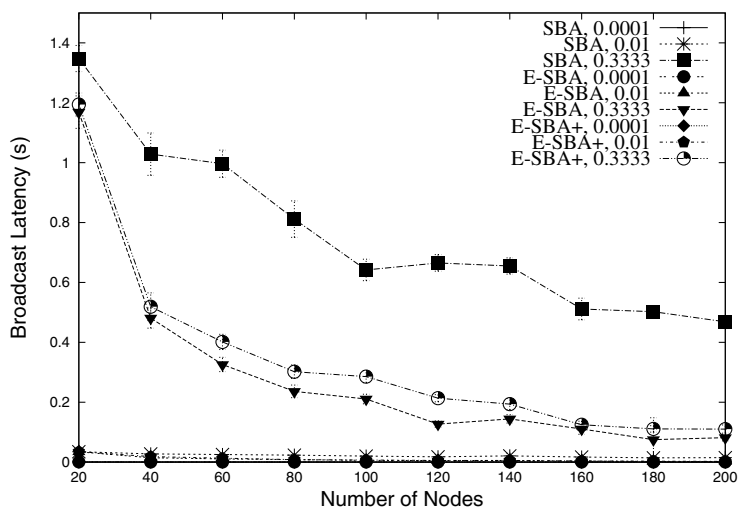

Fig. 4. Broadcast Latency vs. Number of Nodes using various $\Delta$ values

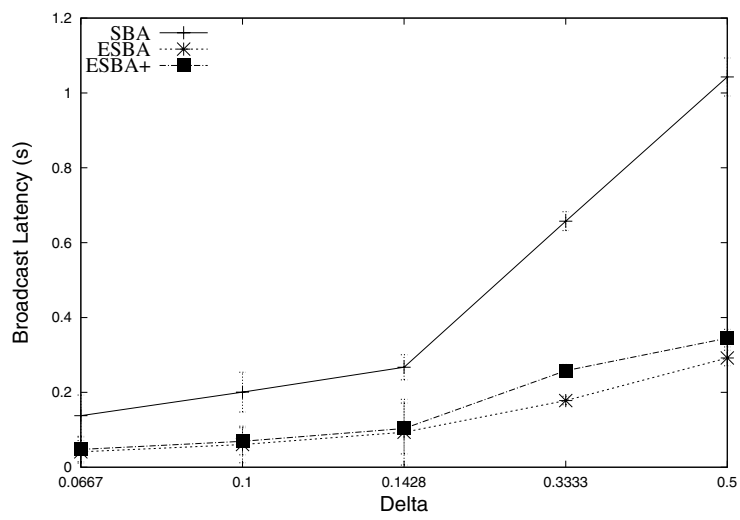

Fig. 5. Selecting $\Delta$ Values for Study 1

The result also indicates that considering 2-hop information, instead of higher degrees, is sufficient in reducing the number of rebroadcasting nodes. Note that all protocols achieved $100 \%$ PDR in this ideal environment.

In Study 2, the three protocols are put to test in mobile environments. The trend in Fig. 7 shows that smaller $\Delta$ results in increasing number of rebroadcasting nodes for all

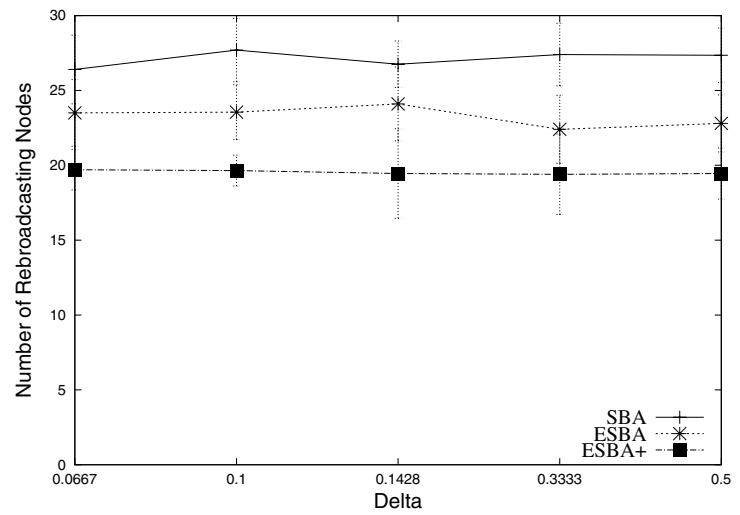

Fig. 6. Effect of Various $\Delta$ Values on Number of Rebroadcasting Nodes 


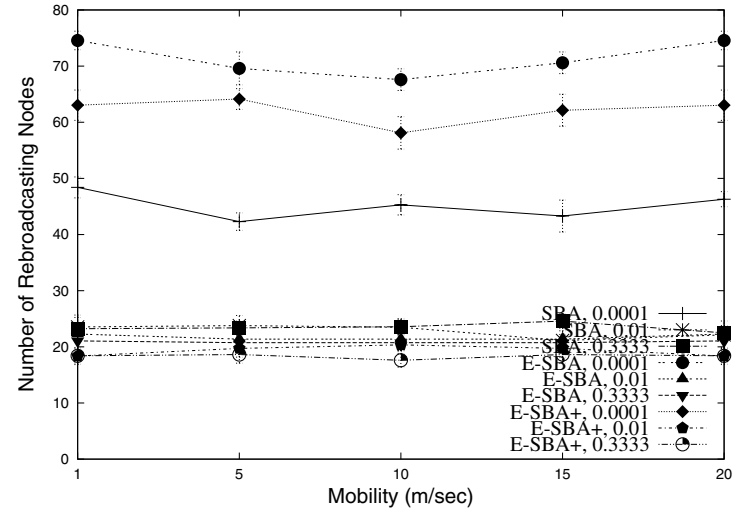

Fig. 7. Number of Rebroadcasting Nodes vs. Mobility

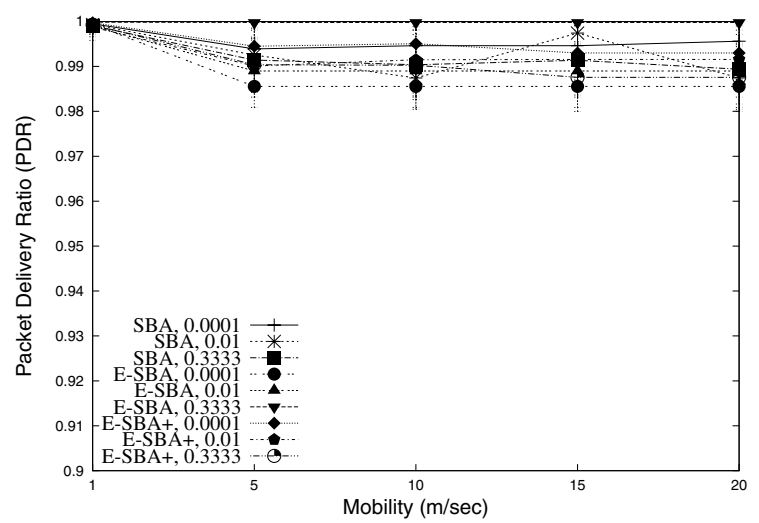

Fig. 8. Packet Delivery Ratio vs. Mobility

node speeds. Since the protocols being studied are based on neighbor-knowledge, any change in the topology will be updated immediately. It is unlikely for a node that receives a data packet from a new neighbor to know any common 1-hop and 2-hop neighbors previously reached. In this case, the node will just rebroadcast the packet. Therefore, all protocols adapt to topology changes by having more redundant rebroadcasting. The proposed protocols performed better than SBA when $\Delta=$ 0.01 and 0.3333 , but not 0.0001 due to lower timer delay. While it is expected that some nodes will not receive all messages, the overall PDR performance of all protocols are at least $98 \%$ as shown in Fig. 8. This shows that the protocols under evaluation effective in adapting to topology change. As shown in Fig. 9, unsurprisingly E-SBA+ has slightly higher broadcast latency than E-SBA due to the timer suppression mechanism. However the increase in delay is insignificant.

\section{CONCLUSION}

This paper proposes E-SBA and E-SBA+ to solve the deficiencies of an existing self-pruning broadcasting protocol SBA. Besides ensuring nodes with more uncovered neighbors rebroadcast first, a timer suppression mechanism to delay the rebroadcasting of nodes with less uncovered nodes was

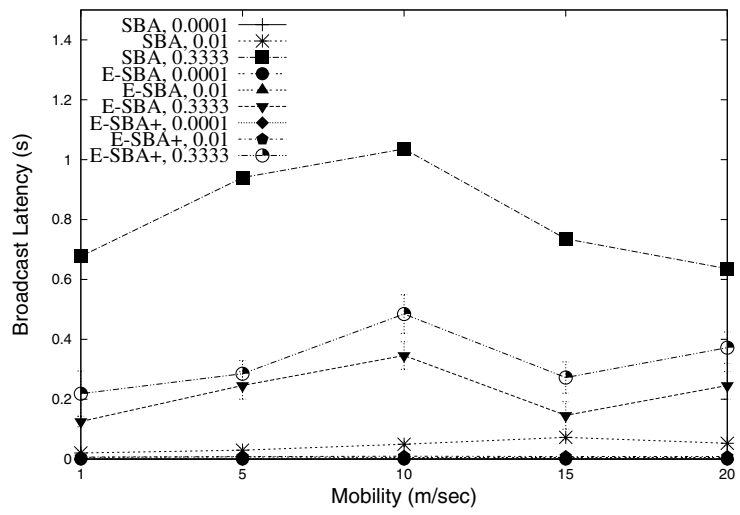

Fig. 9. Broadcast Latency vs. Mobility

designed. Extensive simulation experiments concluded that ESBA and E-SBA+ are effective in achieving the primary goals of reducing the number of rebroadcasting nodes and ensuring high packet delivery ratio.

\section{ACKNOWLEDGEMENT}

The authors thank Dr Tracy Camp and her research group at Toilers group at Colorado School of Mines for sharing the source codes used in this paper.

\section{REFERENCES}

[1] W. Peng and X. C. Lu, "On the Reduction of Broadcast Redundancy in Mobile Ad Hoc Networks," in Proc. of MOBIHOC, 2000.

[2] Y. C. Tseng, S. Y. Ni, Y. S. Chen, and J. P Sheu, "The Broadcast Storm Problem in a Mobile Ad Hoc Network," Wireless Networks, vol. 8, no. 2-3, pp. 153-167, Mar. 2002.

[3] Y C. Tseng, S. Y. Ni, and E. Y. Shih, "Adaptive Approaches to Relieving Broadcast Storms in a Wireless Multihop Mobile Ad Hoc Network," IEEE Trans. on Computers, vol. 52, no. 5, pp. 545-557, May 2003.

[4] C. E. Perkins, E. M. Royer, and I. Chakeres, "Ad Hoc On Demand Distance Vector (AODV) Routing," IETF Internet Draft, draft-perkinsmanet-aodvbis-00.txt, Oct. 2003 (Work in Progress).

[5] Z. J. Haas, M. R. Pearlman, and P. Samar, "The Zone Routing Protocol (ZRP) for Ad Hoc Networks," IETF Internet Draft, draft-ietf-manetzone-zrp-04.txt, July 2002 (Work in Progress).

[6] D. B. Johnson, D. A. Maltz, and Y. C. Hu, "The Dynamic Source Routing Protocol for Mobile Ad Hoc Networks (DSR)," IETF Internet Draft, draft-ietf-manet-dsr-10.txt, july 2004 (Work in Progress).

[7] F. Dai and J. Wu, "Performance Analysis of Broadcast Protocols in Ad Hoc Networks Based on Self-Pruning," IEEE Trans. on Parallel and Distributed Computing, vol. 15, no. 11, pp. 1027-1040, Nov. 2004.

[8] M. Q. Rieck, S. Pai, and S. Dhar, "Distributed routing algorithms for wireless ad hoc networks using d-hop connected dominating sets," in Proc. of HPC-ASIA, pp. 443450, Dec. $2002 \mathrm{f}$.

[9] J. Sucec and I. Marsic, "An efficient distributed network-wide broadcast algorithm for mobile ad hoc networks," CAIP Technical Report 248, Rutgers University, Sep. 2000.

[10] I. Stojmenovic, M. Seddigh, and J. Zunic, "Dominating sets and neighbor elimination based broadcastig algorithms in wireless networks," IEEE Trans. on Parallel and Distributed Systems, vol. 13, no. 1, pp. 1425, Jan. 2002.

[11] B. Williams and T. Camp, "Comparison of Broadcasting Techniques for Mobile Ad Hoc Networks," in Proc. of MOBIHOC, 2002.

[12] K. Fall and K. Varadhan, "The ns manual," The VINT Project, http://www.isi.edu/nsnam/ns/doc/, Nov. 2008.

[13] W. Navidi and T. Camp, "Stationary Distributions for the Random Waypoint Mobility Model," IEEE Trans. On Mobile Computing, vol. 3, no. 1, pp. 99-108, Jan-Mar 2004. 